\title{
Exploring binary-neutron-star-merger scenario of short-gamma-ray bursts by gravitational-wave observation
}

\author{
Kenta Kiuchi, ${ }^{1}$ Yuichiro Sekiguchi, ${ }^{2}$ Masaru Shibata, ${ }^{3}$ and Keisuke Taniguchi ${ }^{4}$ \\ ${ }^{1}$ Department of Physics, Waseda University, 3-4-1 Okubo, Shinjuku-ku, Tokyo 169-8555, Japan \\ ${ }^{2}$ Division of Theoretical Astronomy/Center for Computational Astrophysics, \\ National Astronomical Observatory of Japan, 2-21-1, Osawa, Mitaka, Tokyo, 181-8588, Japan \\ ${ }^{3}$ Yukawa Institute for Theoretical Physics, Kyoto University, Kyoto, 606-8502, Japan \\ ${ }^{4}$ Department of Physics, University of Wisconsin-Milwaukee, P.O. Box 413, Milwaukee, Wisconsin 53201
}

(Dated: September 8, 2018)

\begin{abstract}
We elucidate the feature of gravitational waves (GWs) from binary neutron star merger collapsing to a black hole by general relativistic simulation. We show that GW spectrum imprints the coalescence dynamics, formation process of disk, equation of state for neutron stars, total masses, and mass ratio. A formation mechanism of the central engine of short $\gamma$-ray bursts, which are likely to be composed of a black hole and surrounding disk, therefore could be constrained by GW observation.
\end{abstract}

PACS numbers: 04.25.D-, 04.30.-w, 04.40.Dg

Introduction.-Coalescence of binary neutron stars (BNSs) is one of the most promising sources for kilometer-size laser interferometric gravitational-wave detectors [1]. It is also a candidate for the central engine of short $\gamma$-ray bursts (GRBs), which emit huge energy $\gtrsim 10^{48}$ ergs in a short time scale $\sim 0.1-1 \mathrm{~s}[2,3]$. According to a standard scenario of GRBs based on the so-called merger scenario, a stellar-mass black hole (BH) surrounded by a hot and massive disk (or torus) should be formed after the merger. Possible relevant processes to extract the energy of this $\mathrm{BH}$-accretion disk system for launching a relativistic jet are neutrino-anti neutrino $(\nu \bar{\nu})$ annihilation [4] and/or magnetically driven mechanisms, so-called Blandford-Znajek process [5]. Studies based on the $\nu \bar{\nu}$ annihilation scenario suggest that an accretion rate of $\dot{M} \gtrsim 0.1 M_{\odot} / \mathrm{s}$ is required to achieve a sufficiently high energy efficiency [6] and that if the disk had a mass $\gtrsim 0.01 M_{\odot}$, it could supply the required energy by neutrino radiation for duration of $\gtrsim 100 \mathrm{~ms}$ [7]. General relativistic magnetohydrodynamic simulations also indicate that if a rapidly rotating $\mathrm{BH}$ is formed, its rotational energy can be extracted by the Blandford-Znajek process to achieve a high energy efficiency [8]. However, the merger scenario has not been proven yet observationally, because the counter part for most of short GRB has not been identified [9]. Gravitational waves (GWs) are much more transparent than electromagnetic waves with respect to scattering with matter, and hence, they can propagate from extremely dense region with negligible scattering. Therefore, GWs can be valuable observable for determining the central engine of GRBs [10] and for exploring its formation process. This fact motivates to study coalescence of BNSs and emitted GWs.

For theoretically studying the late inspiral, merger, and ringdown phases of BNSs, numerical relativity is the unique approach. Simulations of BNS merger 11 13 suggest that (i) if the total mass of the BNS is smaller than a threshold mass $\left(M_{\mathrm{thr}}\right)$ a hypermassive neutron star (HMNS) sustained by rapid and differential rotation is formed after the merger and will survive for more than $\sim 100 \mathrm{~ms}$ [11], and (ii) otherwise, a BH is formed after the merger in a dynamical time scale $\sim 1 \mathrm{~ms}$. In the former case, GW emission [11] or magnetic-field effect such as the magnetorotational instability [14] or neutrino radiation [15] will play an essential role in the subsequent evolution of the HMNS, which will eventually collapse to a $\mathrm{BH}$. Because of the complexity, it will not be an easy task to theoretically clarify the formation process of the $\mathrm{BH}$ and gravitational waveforms as well as to accurately predict the launching mechanism of short GRBs. By contrast, in the later case, the formation process of a $\mathrm{BH}$ and surrounding disk is primarily determined by the general relativistic hydrodynamics, and magnetic field or neutrino radiation will play a minor role. Reliable theoretical prediction of merger process and gravitational waveforms by numerical relativity is feasible in this case. Motivated by this idea, we systematically performed numerical relativity simulations to clarify the relation between possible formation process of GRB's central engine (a system composed of a $\mathrm{BH}$ and surrounding disk) and gravitational waveforms, and suggest that GW observation could constrain the formation process for the central engine of short GRBs.

Method and initial models.- Formulation and numerical schemes for solving Einstein's equations, hydrodynamics, and other techniques such as GW extraction are essentially the same as those in Ref. [13], to which the reader may refer for details. For modeling the EOS of neutron stars, we adopt the Akmal-PandharipandeRavenhall (APR) [16], Skyrme-Lyon (SLy) [17], and Friedman-Pandharipande-Skyrme (FPS) [18] EOSs for zero-temperature part. Shock-heating effect, although it gives only minor contribution, is mimicked by $\Gamma$-law EOS with $\Gamma=2$. BNSs of the irrotational velocity field and zero-temperature in quasiequilibrium circular orbits are prepared as initial conditions, which are computed 


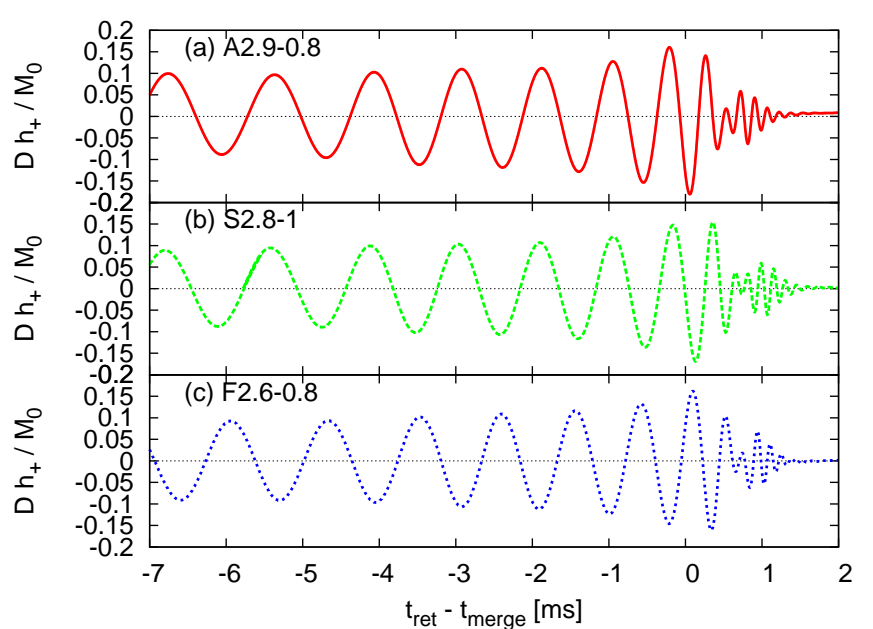

FIG. 1: + modes of GWs for (a) A2.9-0.8, (b) S2.8-1, and (c) F2.6-0.8. $D$ is the distance from the source to the observer, who is located along the axis perpendicular to the orbital plane. $t_{\text {merge }}$ denotes approximate time at the onset of the merger.

by the LORENE library codes [19]. For all the models, the initial orbital separation is large enough that the BNSs spend about 4 orbits in the inspiral phase before the merger. Grid structure and resolution are approximately the same as in Ref. [13]; the major diameter of the neutron stars is covered by about 80 uniform grids and outer boundaries of numerical domain are located at about $1.2 \lambda_{0}$ where $\lambda_{0}$ is initial gravitational wavelength. Total mass, $m_{0}$, is chosen to be large enough that a $\mathrm{BH}$ is formed in a dynamical time scale $(\sim 1 \mathrm{~ms})$ after the onset of the merger. This can be achieved for $m_{0}>M_{\mathrm{thr}}$, where $M_{\mathrm{thr}} \approx 2.8-2.9 M_{\odot}$ for the APR, $\approx 2.7-2.8 M_{\odot}$ for the SLy, and $\approx 2.5-2.6 M_{\odot}$ for the FPS EOS, respectively [11. Total mass and mass ratio are chosen in the range of $2.9 M_{\odot} \leq m_{0} \leq 3.1 M_{\odot}, 0.8 \leq \nu \equiv m_{1} / m_{2} \leq 1$ for the APR, $2.8 M_{\odot} \leq m_{0} \leq 3.0 M_{\odot}, \nu=1$ for the SLy, and $2.6 M_{\odot} \leq m_{0} \leq 2.8 M_{\odot}, \nu=0.8$ and 1 for the FPS EOS. $m_{1}$ and $m_{2}\left(m_{2} \geq m_{1}\right)$ denote the gravitational mass of two neutron stars in isolation and $m_{0}=m_{1}+m_{2}$. We name the models according to the EOS, $m_{0}$, and $\nu$; e.g., A2.9-0.9 is modeled by the APR EOS with $m_{0}=2.9 M_{\odot}$ and $\nu=0.9$.

Results.-We investigate the relation between GWs and possible formation process of a BH-disk system in a wide range of parameter space. First of all, we briefly review a typical coalescence process of BNSs collapsing dynamically to a BH (see Ref. [13] in details). During the inspiral phase, the BNSs adiabatically evolve gradually decreasing its orbital separation due to the gravitational radiation reaction. GWs in this phase are characterized by a chirp signal (see the waveform for $t_{\text {ret }}-t_{\text {merge }} \lesssim 0$ in Fig. 11). After the binary separation becomes $\lesssim 3$ neutron star radii, the merger sets in. For $\nu \approx 1$, a high-density region with density $>10^{15} \mathrm{~g} / \mathrm{cm}^{3}$ is sub-

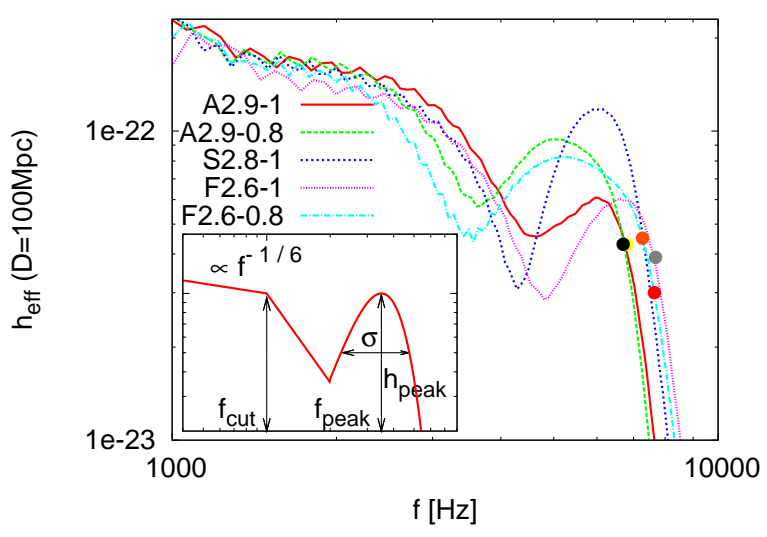

FIG. 2: Effective amplitude for models A2.9-1, A2.9-0.8, S2.81, F2.6-1, and F2.6-0.8. The filled circles denote the QNM frequency of the formed BHs. The small panel shows a schematic figure of the GW spectrum.

sequently formed at the center, and then, collapses to a $\mathrm{BH}$ within $\sim 1 \mathrm{~ms}$. During this merger process, small spiral arms, composed of two symmetric arms, are formed around the $\mathrm{BH}$ but they are eventually swallowed by the $\mathrm{BH}$. The final outcome is a rotating $\mathrm{BH}$ nearly in a vacuum state. For $\nu \lesssim 0.9$, the less massive star is tidally deformed in a close orbit and disrupted just after the onset of the merger. The outer part of the tidally disrupted neutron-star matter forms an asymmetric spiral arm which is more massive and widely-spread than that for the equal-mass case. Although the central highdensity region, formed at the onset of the merger, collapses to a $\mathrm{BH}$, the large spiral arm subsequently forms an accretion disk around the $\mathrm{BH}$ because it has angular momentum large enough to escape from the capture by the BH. GWs in the merger phase are characterized by the short-term burst-type waves $\left(0 \lesssim t_{\text {ret }}-t_{\text {merge }} \lesssim 1 \mathrm{~ms}\right.$ in Fig. (1) and after the BH formation, quasinormal modes (QNM) are excited $\left(t_{\text {ret }}-t_{\text {merge }} \gtrsim 1 \mathrm{~ms}\right.$ in Fig. 1). Figure1, which plots GWs for A2.9-0.8, S2.8-1, and F2.6-0.8 and shows typical waveforms for the BNS merger, illustrates features mentioned above.

GW spectrum more clearly reflects dynamics of the merger process. Figure 2 plots an effective amplitude of GWs as a function of the frequency, $h_{\mathrm{eff}}(f)$, for models A2.9-0.8, A2.9-1, S2.8-1, F2.6-1, and F2.6-0.8. The effective amplitude is defined by $h_{\text {eff }} \equiv \tilde{h}(f) f m_{0} / D$ with $\tilde{h}(f)$ being the Fourier transform of $h_{+}+i h_{\times}$. We assume that the distance from the observer to the source is $D=100$ $\mathrm{Mpc}$, because more than one event per year is predicted for such a large distance [20]. Note that GW frequency increases as the orbital separation decreases and as the compactness of the merged object increases: GWs with $f \lesssim 1 \mathrm{kHz}, 1 \lesssim f \lesssim 3 \mathrm{kHz}$, and $f \gtrsim 3 \mathrm{kHz}$ are emitted in the inspiral phase, early merger, and late merger phases, respectively. 


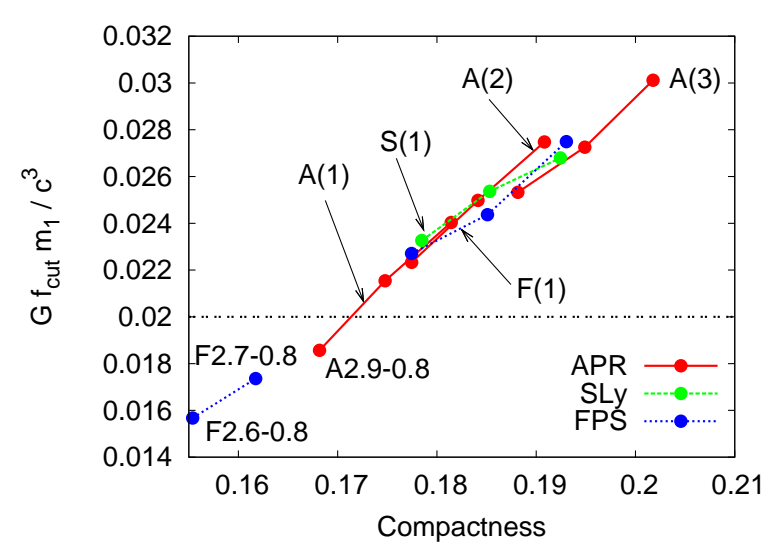

FIG. 3: $\mathrm{G} f_{\text {cut }} m_{1} / \mathrm{c}^{3}$ as a function of compactness of less massive binary component for all the models. Sequence A(1) represents the models A2.9-0.8, A3-0.8, and A3.1-0.8 from left to right. In a similar way, $\mathrm{A}(2)$ is a sequence of $\mathrm{A} 2.9-0.9, \mathrm{~A} 3-0.9$, and A3.1-0.9, A(3) is A2.9-1, A3-1, and A3.1-1, S(1) is S2.8-1, S2.9-1, and S3-1, and F(1) is F2.6-1, F2.7-1, and F2.8-1. The models below the horizontal line of $\mathrm{G} f_{\text {cut }} m_{1} / \mathrm{c}^{3}=0.02$ can produce disks of mass $\geq 0.01 M_{\odot}$.

The effective amplitude gradually decreases for $f \lesssim 3$ $\mathrm{kHz}$ according to $\propto f^{-n}$ with $n \approx 1 / 6$ which is the typical number for the inspiral phase, and for $f \gtrsim f_{\text {cut }} \approx 2.5-4$ $\mathrm{kHz}$, its gradient becomes steep (see the small panel in Fig. 2 for $\left.f \gtrsim f_{\text {cut }}\right)$. For $f \lesssim f_{\text {cut }}$, the merged object has a binary-like structure (i.e., there exist two density maxima) which enhances emissivity of GWs, whereas at $f \sim f_{\text {cut }}$, such structure is disrupted, resulting in a quick decrease of the GW amplitude. A hump, which appears for $4 \lesssim f \lesssim 7 \mathrm{kHz}$ with the peak frequency $f_{\text {peak }} \sim 5-6$ $\mathrm{kHz}$, reflects the formation and evolution of the spiral arms orbiting the central object. For $f>f_{\text {peak }}, h_{\text {eff }}$ exponentially decreases; this is the typical feature for the spectrum associated with a QNM ring-down of the formed BH. These spectrum features are qualitatively universal as shown in Fig. 2. However, the spectrum shape depends quantitatively on the EOS, $m_{0}$, and $\nu$.

The spectrum shape for $f \gtrsim f_{\text {cut }}$ is characterized by $f_{\text {cut }}, f_{\text {peak }}$, and peak amplitude and width of the hump $\left(h_{\text {peak }}\right.$ and $\left.\sigma\right)$, for which a schematic figure is described in the small panel of Fig. 2. We perform a fitting procedure to extract these characteristic quantities from the spectrum. As the first step, the spectrum is divided into two parts; one is for $f \leq f_{\mathrm{d}}$, inspiral and early merger phases, and the other is for $f \geq f_{\mathrm{d}}$, the merger and ringdown phases. $f_{\mathrm{d}}$ is typically chosen as $4 \mathrm{kHz}$. For $f \leq f_{\mathrm{d}}$, we fit the spectrum by $f^{-1 / 6} h_{0} /\left[1+\exp \left\{\left(f-f_{\text {cut }}\right) / \Delta f\right\}\right]$. For $f \geq f_{\mathrm{d}}$, we adopt the Gaussian distribution $h_{\text {peak }} \exp \left[-\left(f-f_{\text {peak }}\right)^{2} / \sigma^{2}\right]$. In the fitting, $f_{\text {cut }}, \Delta f, h_{0}, h_{\text {peak }}, f_{\text {peak }}$, and $\sigma$ are determined by the $\chi$-square fitting [21].

Figures 3 plots $\mathrm{G} f_{\text {cut }} m_{1} / \mathrm{c}^{3}$ as a function of compactness of less massive companion defined by $\mathrm{Gm}_{1} / c^{2} R$

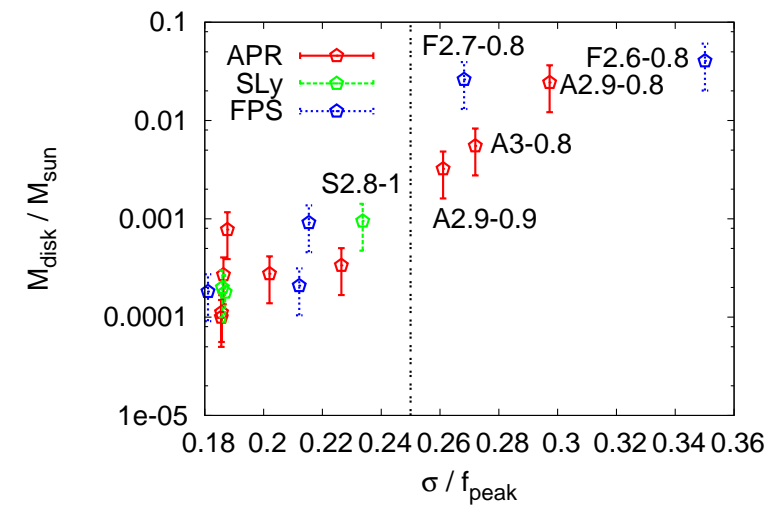

FIG. 4: Disk mass as a function of $\sigma / f_{\text {peak }}$ for all the models.

where $R$ is its circumferential radius. $\mathrm{G} f_{\text {cut }} m_{1} / c^{3}$ correlates strongly with $\mathrm{G} m_{1} / c^{2} R$. $\mathrm{G} f_{\text {cut }} m_{1} / c^{3}$ depends also on $m_{0}$; for the larger value of $m_{0}$, it is larger for a given EOS and $\nu$. Note that larger values of $m_{0}$ and $\nu$ result in smaller values of disk mass (see Fig. 4). Thus, $f_{\text {cut }}$ is an indicator that shows the occurrence of the tidal disruption and possible disk formation. (We note that to obtain $f_{\text {cut }} m_{1}$, we have to determine $m_{1}$ from the gravitationalwave signal in the inspiral phase 22]. It is also worthy to note that $R$ may be determined, if $f_{\text {cut }}$ is measured and $m_{1}$ is determined; see Ref. [23] for the related topic.)

Figures 4 plots the mass of disk surrounding a $\mathrm{BH}$ as a function of $\sigma / f_{\text {peak }}$. This shows that the disk mass has a positive correlation with $\sigma / f_{\text {peak }}$. On the other hand, we find that the disk mass does not have a clear correlation with $h_{\text {peak }}$. The disk mass also correlates with $m_{0}-M_{\text {thr }}$ and $\nu: m_{0}-M_{\text {thr }} \lesssim 0.2 M_{\odot}$ and $\nu \lesssim 0.8$ appear to be necessary for producing a massive disk with $\geq 0.01 M_{\odot}$ (see A2.9-0.8, F2.6-0.8, and F2.7-0.8). The disk mass for other models which do not satisfy this condition is $10^{-3}$ $10^{-5} M_{\odot}$ [11, 13]. Combination of Figs. 3 and 4 also proposes that a necessary condition for producing a disk of mass $\gtrsim 0.01 M_{\odot}$ is $\mathrm{G} f_{\text {cut }} m_{1} / c^{3} \leq 0.02$ and $\sigma / f_{\text {peak }} \gtrsim$ 0.25 .

The frequency of GWs in the merger phase is slightly outside the most sensitive frequency band of the first and second generation detectors such as LIGO and advanced LIGO $(10-1 \mathrm{kHz})$, because the values of $f_{\text {cut }}$ and $f_{\text {peak }}$ are $\sim 2.5-7 \mathrm{kHz}$. Nevertheless, the amplitude at $f=f_{\text {cut }}$ and $f_{\text {peak }}$ is not extremely small for a hypothetical distance $D=100 \mathrm{Mpc}$. If the proposed third generation detectors such as Einstein telescope become available with a special design for high-frequency sensitivity, these quantities may be measured. Our results indicate that the formation and evolution processes of a $\mathrm{BH}$ and surrounding disk are imprinted in GW spectrum. Also, GWs are the unique observable which directly carries information of the central region of GRBs. Thus, it is worthy to explore methods for extracting physical information on the 
merger of BNSs collapsing to a $\mathrm{BH}$ from observed GWs, which may clarify the formation process of short GRBs.

Constraining the merger process by $G W$ observation.As mentioned in Introduction, the merger hypothesis for the central engine of short GRBs requires that the mass of disk around a $\mathrm{BH}$ is greater than $\sim 0.01 M_{\odot}$. Our numerical results show that disk mass correlates strongly with $\sigma / f_{\text {peak }}$ and $\mathrm{G} f_{\text {cut }} m_{1} / c^{3}$ irrespective of EOS. Assuming that GWs from BNSs are frequently observed, and also, GWs and short GRB are observed simultaneously for several events in the future, we propose a strategy for exploring the merger hypothesis by analyzing GWs. As a first step, we should determine $m_{0}, \nu$, $f_{\text {cut }}, f_{\text {peak }}$, and $\sigma$ for each event: The values of $m_{0}$ and $\nu$ will be determined from the inspiral waveform using the matched filtering technique [22], and $f_{\text {cut }}, f_{\text {peak }}$, and $\sigma$ be from the merger waveform. As a second step, we should infer the disk mass from $\mathrm{G} f_{\text {cut }} m_{1} / c^{3}$ and $\sigma / f_{\text {peak. If }}$ the conditions of $\mathrm{G} f_{\text {cut }} m_{1} / c^{3} \leq 0.02$ and $\sigma / f_{\text {peak }} \gtrsim 0.25$ are always satisfied for events in which GWs and short GRB are simultaneously observed, the merger hypothesis will be strongly supported. Because a small value of $\mathrm{G} f_{\text {cut }} m_{1} / c^{3}$ (for the typical mass of neutron stars $1.3-$ $\left.1.5 M_{\odot}\right)$ implies that mass ratio is much smaller than unity, we could conclude that the origin of short GRBs would be the merger of unequal-mass BNSs. By contrast, if simultaneous detections occur irrespective of the values of $\mathrm{G} f_{\text {cut }} m_{1} / c^{3}$ and $\sigma / f_{\text {peak }}$, this does not agree with our numerical results, because these should correlate with the disk mass. In such case, we have to conclude that an unknown mechanism plays a role for producing the GRBs.

Our numerical results indicate that massive disk is not formed for $\nu \approx 1$ or $m_{0}>3 M_{\odot}$ for any EOS. This implies that the merger of BNS collapsing to a $\mathrm{BH}$ does not always produce GRBs. For such case, only GWs will be observed. This suggests another test for the merger scenario.

Finally, a comment is given for the case that an HMNS is formed. Because the HMNS should eventually collapse to a $\mathrm{BH}$ via gravitational radiation [11] or angular momentum transport by magnetohydrodynamic effect [14] or neutrino cooling, the HMNS formation scenario can be an alternative in the merger hypothesis. In the HMNS formation, the GW spectrum for the merger phase is significantly different from that in the $\mathrm{BH}$ formation because quasiperiodic GWs emitted by a quasiperiodic rotation of the HMNS is likely to produce sharp peaks in the spectrum and characterize the spectrum [11]. In this case, detecting these peaks of GWs will play a crucial role for confirming the merger hypothesis.

Acknowledgments. - Numerical computations were performed on XT4 at the Center for Computational Astrophysics in NAOJ and on NEC-SX8 at YITP in Kyoto University. This work was supported by Grant-in-Aid for Scientific Research (21340051) and for Scientific Research on Innovative Area (20105004) of the Japanese MEXT, by NSF Grant PHY-0503366, and by Grant-in-Aid of the Japanese Ministry of Education, Science, Culture, and Sport (21018008,21105511).

[1] B. C. Barish and R. Weiss, Phys. Today 52N10, 44 (1999); S. Hild, Class. Quant. Grav. 23, S643 (2006); F. Acernese et al. [VIRGO Collaboration], Class. Quant. Grav. 19, 1421 (2002); M. Ando et al. (the TAMA collaboration), Phys. Rev. Lett. 86, 3950 (2001).

[2] R. Narayan, B. Paczynski and T. Piran, Astrophys. J. 395, L83 (1992).

[3] B. Zhang and P. Meszaros, Int. J. Mod. Phys. A 19, 2385 (2004).

[4] T. Piran, Rev. Mod. Phys. 76, 1143 (2005).

[5] R. D. Blandford, and R. L. Znajek, Mon. Not. Roy. Astron. Soc. 179, 433 (1977).

[6] E.g., R. Popham, S. E. Woosley, and C. Fryer, Astrophys. J. 518, 356 (1999)

[7] E.g., S. Setiawan, M. Ruffert, and H.-Th. Janka, Mon. Not. R. Astron. Soc. 352, 753 (2004).

[8] E.g., J. C. McKinney and C. F. Gammie, Astrophys. J. 611, 977 (2004).

[9] E.g., D. B. Fox et al., Nature 437, 845 (2005).

[10] S. Kobayashi and P. Meszaros, Astrophys. J. 589, 861, (2003).

[11] M. Shibata, K. Taniguchi and K. Uryu, Phys. Rev. D 68, 084020 (2003): Phys. Rev. D 71, 084021 (2005): M. Shibata, Phys. Rev. Lett. 94, 201101 (2005): M. Shibata and K. Taniguchi, Phys. Rev. D 73, 064027 (2006).

[12] L. Baiotti, B. Giacomazzo and L. Rezzolla, Phys. Rev. D 78, 084033 (2008).

[13] K. Kiuchi, Y. Sekiguchi, M. Shibata and K. Taniguchi, Phys. Rev. D 80, 064037 (2009).

[14] M. D. Duez et al., Phys. Rev. D 73, 104015 (2006): M. D. Duez et al., Phys. Rev. Lett. 96, 031101 (2006): M. Shibata et al., Phys. Rev. Lett. 96, 031102 (2006).

[15] R. Oechslin and H. T. Janka, Phys. Rev. Lett. 99, 121102 (2007).

[16] A. Akmal, V. R. Pandharipande, and D. G. Ravenhall, Phys. Rev. C 58, 1804 (1998).

[17] F. Douchin and P. Haensel, A\&A, 380, 151 (2001).

[18] B. Friedman, abd V. R. Pandharipande, 1981, Nuclear Physics A, 361, 502; V. R. Pandharipande and D. G. Ravenhall, 1989, NATO ASIB Proc. 205; Nuclear Matter \& Heavy Ion Collisions, 103 .

[19] E. Gourgoulhon, et al., Phys. Rev. D 63, 064029 (2001); K. Taniguchi and E. Gourgoulhon, Phys. Rev. D 66, 104019 (2002), Phys. Rev. D 68, 124025 (2003); LORENE website: http://www.lorene.obspm.fr/.

[20] V. Kalogera et al., Phys. Rep. 442, 75 (2007).

[21] W. Press, B. P. Flannery, S. Teukolosky and W. T. Vetterling, Numerical Recipies in $C$ (Cambridge University Press, Cambridge, England, 1986).

[22] C. Cutler and E.E. Flanagan, Phys. Rev. D 49, 2658 (1994): We note that for binaries composed of NSs which cannot have a large spin, it will be possible to determine each mass with a good accuracy.

[23] J.S. Read, et al., Phys. Rev. D 79, 124033 (2009) 